Review Article

\title{
Constrained extension of linear operators, approximation, and the moment problem
}

\author{
Octav Olteanu*
}

Department of Mathematics and Informatics, University Politehnica of Bucharest, Bucharest 060042, Romania

(Received: 19 November 2021. Received in revised form: 16 December 2021. Accepted: 18 December 2021. Published online: 23 December 2021.)

(C) 2021 the author. This is an open access article under the CC BY (International 4.0) license (www.creativecommons.org/licenses/by/4.0/).

\begin{abstract}
Some Hahn-Banach-extension results for linear operators are reviewed. Several direct applications of these extension results to the existence of a solution for Markov moment problems are pointed out. A variant of the Mazur-Orlicz theorem in concrete spaces is also proved. Moreover, polynomial-approximation results on unbounded subsets and their applications to the existence and uniqueness of the solutions for the full Markov moment problem on $\mathbb{R}^{n}$ and on $\mathbb{R}_{+}^{n}$ are reviewed. The multi-dimensional case is considered and the one-dimensional case follows as a consequence. Finally, the truncated moment problem is briefly discussed.
\end{abstract}

Keywords: extension of linear operators; sandwich constraints; full Markov moment problem; existence of a solution; polynomial approximation; unbounded subsets; uniqueness.

2020 Mathematics Subject Classification: 46A22, 41A10.

\section{Introduction}

Studying and proving extension results for linear functionals and operators are usually motivated by (i) proving existence of solutions for some moment problems (see [1,2,5,6,11,13,14,21-26,28,30,31]), (ii) sub-differentiability of convex functions and convex operators (see $[17,18,20]$ ), (iii) decomposition of linear operators acting on ordered vector spaces (the codomain being an order complete vector lattice) as a difference of two positive linear operators, the first one being dominated by a given convex operator (see [17, 19, 20]), (iv) the Krein-Milman theorem and some other problems. The Krein-Milman theorem has applications also to representation theorems, convex optimization and some other fields of analysis (see $[4,5$, 18]). Many results on the moment problem, not necessarily or explicitly using extension of linear functional and operators, have been published in $[3,4,8-10,12,15,16,28,32,33]$. Old results of the present author published in [19-21] have found several applications specified in $[2,17,22,23,25]$. Also, several polynomial-approximation results on unbounded subsets reviewed in the recent paper [24] have found applications [25, 26]. Density theorems in a different framework were published in [27]. In present paper, the following notations are used: $\mathbb{N}=\{0,1,2, \ldots\}$ is the set of all nonnegative integers, $\mathcal{P}$ is the real vector space of all polynomial functions with real coefficients. If $F \subseteq \mathbb{R}^{n}$ is a closed unbounded subset, then denote by $C_{c}(F)$ the vector space of all real-valued continuous compactly supported functions defined on $F$. Take $\mathbb{R}_{+}=[0,+\infty)$. Basic notions and results in analysis related to this paper can be found in the monographs $[1,4,7,13,18,29,31]$.

The remaining part of this paper is organized as follows. Section 2 is devoted to general extension results for linear operators. A variant of the Mazur-Orlicz theorem is also considered in Section 2. In Section 3, some direct applications of the results of Section 2 are specified. Section 4 is concerned with a polynomial approximation on unbounded subsets and its applications to the existence and uniqueness of the solution for some classical full Markov moment problems. Section 5 is devoted to some comments and remarks on the truncated moment problem. Section 6 concludes the paper.

\section{On constrained extensions for linear operators}

One of the earlier results on extension of linear operators refers to the existence of a positive linear extension for a positive linear operator defined on a majorizing subspace of an ordered vector space. The codomain space is an order complete vector space. Here, the constraint is the positivity of the extension on the positive cone of the domain space. Let $X_{1}$ be an ordered vector space whose positive cone $X_{1,+}$ is generating $\left(X_{1}=X_{1,+}-X_{1,+}\right)$. Recall that in such an ordered vector space $X_{1}$, a vector subspace $S$ is said to be a majorizing subspace if for any $x \in X_{1}$ there exists $s \in S$ such that $x \leq s$. Following

\footnotetext{
*E-mail address: octav.olteanu50@gmail.com
} 
is a significant example of a majorizing subspace. Let $F \subseteq \mathbb{R}^{n}$ be a closed unbounded subset and $1 \leq \alpha<+\infty$. Let $\nu$ be a positive regular Borel measure on $F$, with finite moments of all orders. Define $X:=L_{\nu}^{\alpha}(F)$ and let $X_{1}$ be the vector subspace of all functions $f \in X$ for which there exists a polynomial $p$ such that $|f| \leq p$ on $F$. Then the subspace $S:=\mathcal{P}$ of all polynomial functions on $F$ is a majorizing subspace of $X_{1}$. The space $X_{1}$ contains $C_{c}(F)$ (the subspace of all continuous compactly supported real functions on $F)$, as well as the subspace $\mathcal{P}\left(p \in \mathcal{P} \Longrightarrow|p|=\sqrt{1 \cdot p^{2}} \leq\left(\left(1+p^{2}\right) / 2\right) \in \mathcal{P}\right)$. The subspace $X_{1}$ is dense in $X$, since it contains $C_{c}(F)$, which is dense in $L_{\nu}^{\alpha}(F)=X$.

Theorem 2.1 (see [14], Theorem 1.2.1). Let $X_{1}$ be an ordered vector space whose positive cone is generating, $X_{0} \subset X_{1} a$ majorizing vector subspace, $Y$ an order complete vector space, $T_{0}: X_{0} \rightarrow Y$ a positive linear operator. Then $T_{0}$ admits a positive linear extension $T: X_{1} \rightarrow Y$.

A much more general form of Theorem 2.1 was proved in [19] (see also [20] for details and completions). In the next theorem, $X$ is a real vector space, $Y$ an order-complete vector lattice, $A, B \subseteq X$ convex subsets, $Q: A \rightarrow Y$ a concave operator, $P: B \rightarrow Y$ a convex operator, $M \subset X$ a vector subspace, $T_{0}: M \rightarrow Y$ a linear operator. All vector spaces and linear operators are considered over the real field.

Theorem 2.2 (see [19], Theorem 1). Assume that $T_{0}(x) \geq Q(x) \forall x \in M \cap A$ and $T_{0}(x) \leq P(x) \forall x \in M \cap B$. The following statements are equivalent:

(a). There exists a linear extension $T: X \rightarrow Y$ of the operator $T_{0}$ such that

$$
\left.T\right|_{A} \geq Q \quad \text { and }\left.\quad T\right|_{B} \leq P .
$$

(b). There exists a convex operator $P_{1}: A \rightarrow Y$ and a concave operator $Q_{1}: B \rightarrow Y$ such that for all

$$
\left(\rho, t, \lambda, a_{1}, a, b_{1}, b, v\right) \in[0,1]^{2} \times(0, \infty) \times A^{2} \times B^{2} \times M,
$$

the following implication holds:

$$
(1-t) a_{1}-t b_{1}=v+\lambda((1-\rho) a-\rho b) \quad \Longrightarrow \quad(1-t) P_{1}\left(a_{1}\right)-t Q_{1}\left(b_{1}\right) \geq T_{0}(v)+\lambda((1-\rho) Q(a)-\rho P(b)),
$$

where the convex subsets $A, B$ are arbitrary, with no restriction on the existence of relative interior points or on their position with respect to the subspace $M$.

Theorem 2.3 (see [19], Theorem 2). Let $X$ be an ordered vector space, $Y$ an order complete vector space, $M \subset X$ a vector subspace, $T_{1}: M \rightarrow Y$ a linear operator, $P: X \rightarrow Y$ a convex operator. The following statements are equivalent:

(a). there exists a positive linear extension $T: X \rightarrow Y$ of $T_{1}$ such that $T \leq P$ on $E$;

(b). it holds that $T_{1}(h) \leq P(x)$ for all $(h, x) \in M \times X$ such that $h \leq x$.

One observes that in the very particular case $E_{+}=\{0\}$, when the order relation on $E$ is the equality then from Theorem 2.3 one obtains Hahn-Banach extension theorem for linear operators dominated by convex operators. Unlike Theorems 2.2 and 2.3, which provide necessary and sufficient conditions for the existence of an extension, the next result gives only sufficient conditions.

Theorem 2.4 (see [20]). Let $X$ be a locally convex space, $Y$ an order complete vector lattice with strong order unit $u_{0}$ and $S \subset X$ a vector subspace. Let $C \subset X$ be a convex subset with the following properties:

(a). There exists a neighborhood $D$ of the origin such that $(S+D) \cap C=\emptyset$ (that is, by definition, $C$ and $S$ are distanced).

(b). $C$ is bounded.

Then for any equicontinuous family of linear operators $\left\{f_{j}\right\}_{j \in J} \subset \mathcal{L}(S, Y)$ and for any $\tilde{y} \in Y_{+} \backslash\{\mathbf{0}\}$, there exists an equicontinuous family $\left\{T_{j}\right\}_{j \in J} \subset \mathcal{L}(X, Y)$ such that

$$
T_{j}(s)=f_{j}(s), s \in S, T_{j}() \geq \tilde{y}, \in C, j \in J .
$$

Moreover, if $D$ is a convex balanced neighbourhood of the origin such that

$$
f_{j}(D \cap S) \subset\left[-u_{0}, u_{0}\right], \quad(S+D) \cap C=\emptyset
$$

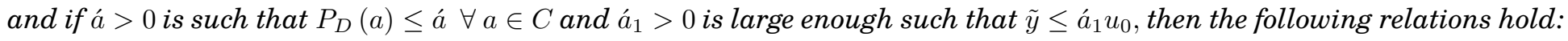

$$
T_{j}(x) \leq\left(1+\dot{a}+\dot{a}_{1}\right) P_{D}(x), \quad x \in X, j \in J,
$$

where $P_{D}$ denotes the gauge (Minkowski functional) attached to $D$. 
Theorem 2.5 (see [21], Theorem 5). Let $X$ be a preordered vector space and $Y$ be an order complete vector space. Let $\left\{x_{j}\right\}_{j \in J}$ and $\left\{y_{j}\right\}_{j \in J}$ be the families of elements in $X$ and $Y$, respectively, and let $P: X \rightarrow Y$ be a sublinear operator. The following statements are equivalent:

(a). There exists a linear positive operator $T: X \rightarrow Y$ such that

$$
T\left(x_{j}\right) \geq y_{j}, j \in J, T(x) \leq P(x), x \in X .
$$

(b). For any finite subset $J_{0} \subset J$ and any $\left\{\lambda_{j}\right\}_{j \in J_{0}} \subset \mathbb{R}_{+}$, the following implication holds

$$
\sum_{j \in J_{0}} \lambda_{j} x_{j} \leq x \in X \Longrightarrow \sum_{j \in J_{0}} \lambda_{j} y_{j} \leq P(x) .
$$

\section{Applications to the Markov moment problem and Mazur-Orlicz theorem}

We start this section with two applications to the abstract moment problem. The first one is another way of writing of Theorem 2.3.

Theorem 3.1 (see [21], Theorem 1). Let $X$ be a preordered vector space, $Y$ an order complete vector lattice, $P: X \rightarrow Y a$ convex operator, $\left\{x_{j}\right\}_{j \in J} \subset X,\left\{y_{j}\right\}_{j \in J} \subset Y$ given families. The following statements are equivalent:

(a). There exists a linear positive operator $T: X \rightarrow Y$ such that

$$
T\left(x_{j}\right)=y_{j}, j \in J, T(x) \leq P(x), x \in X .
$$

(b). For any finite subset $J_{0} \subseteq J$, and any $\left\{\lambda_{j} ; j \in J_{0}\right\} \subseteq \mathbb{R}$, it holds that

$$
\sum_{j \in J_{0}} \lambda_{j} x_{j} \leq x \in X \Longrightarrow \sum_{j \in J_{0}} \lambda_{j} y_{j} \leq P(x) .
$$

In addition, if we assume that $P$ is isotone $(u \leq v \Rightarrow P(u) \leq P(v))$, the assertions (a) and (b) are equivalent to (c), where:

(c). For any finite subset $J_{0} \subset J$ and any $\left\{\lambda_{j}\right\}_{j \in J_{0}} \subset \mathbb{R}$, the following inequality holds:

$$
\sum_{j \in J_{0}} \lambda_{j} y_{j} \leq P\left(\sum_{j \in J_{0}} \lambda_{j} x_{j}\right) .
$$

Theorem 3.2 (see [21], Theorem 4). Let $X$ be an ordered vector space and $Y$ be an order complete vector lattice. Also, let $\left\{\varphi_{j}\right\}_{j \in J} \subset X,\left\{y_{j}\right\}_{j \in J} \subset Y$ be two given arbitrary families and $T_{1}, T_{2} \in L(X, Y)$ be two linear operators. The following statements are equivalent:

(a). There is a linear operator $T \in L(X, Y)$ such that

$$
T_{1}(x) \leq T(x) \leq T_{2}(x) \quad \forall x \in X_{+}, T\left(\varphi_{j}\right)=y_{j} \forall j \in J .
$$

(b). For any finite subset $J_{0} \subset J$ and any $\left\{\lambda_{j} ; j \in J_{0}\right\} \subset \mathbb{R}$, the following implication holds

$$
\left(\sum_{j \in J_{0}} \lambda_{j} \varphi_{j}=\psi_{2}-\psi_{1}, \psi_{1}, \psi_{2} \in X_{+}\right) \Longrightarrow \sum_{j \in J_{0}} \lambda_{j} y_{j} \leq T_{2}\left(\psi_{2}\right)-T_{1}\left(\psi_{1}\right) .
$$

If $X$ is a vector lattice, then each of the assertions ( $a$ ) and (b) is equivalent to the following assertion:

(c). $T_{1}(w) \leq T_{2}(w)$ for all $w \in X_{+}$and for any finite subset $J_{0} \subset J$ and for all $\left\{\lambda_{j} ; j \in J_{0}\right\} \subset \mathbb{R}$, we have

$$
\sum_{j \in J_{0}} \lambda_{j} y_{j} \leq T_{2}\left(\left(\sum_{j \in J_{0}} \lambda_{j} \varphi_{j}\right)^{+}\right)-T_{1}\left(\left(\sum_{j \in J_{0}} \lambda_{j} \varphi_{j}\right)^{-}\right) .
$$

The next results provide applications of some of the preceding theorems to concrete spaces. We recall that a sequence $\left(u_{n}\right)_{n \geq 0}$ in an ordered vector space $Y$ is called nonnegative with respect to an interval $\mathbb{I} \subseteq \mathbb{R}$ if for any $n \in \mathbb{N}$, and any coefficients $\lambda_{j}, j=0,1, \ldots, n$, we have

$$
\lambda_{0}+\lambda_{1} t+\cdots+\lambda_{n} t^{n}=0 \quad \forall t \in \mathbb{I} \Longrightarrow \lambda_{0} u_{0}+\lambda_{1} u_{1}+\cdots \lambda_{n} u_{n} \in Y_{+} .
$$


Theorem 3.3 (see [22], Theorem 31). Let

$$
\begin{gathered}
0<b \in \mathbb{R}, X=C([0, b]), \varphi_{j}(t)=t^{j}, j \in \mathbb{N}, j \geq 1, t \in[0, b], \\
\left\{\psi_{k}\right\}_{k=0} \subset X,\left\|\psi_{k}\right\| \leq 1, \psi_{0}=\mathbf{1}, \psi_{k}(0)=1, k \in \mathbb{N}, k \geq 1 .
\end{gathered}
$$

Let $Y$ be an order complete vector lattice with strong order unit $u_{0}$ and $\left(y_{n}\right)_{n \geq 1}$ a sequence in $Y$ such that $\left(u_{0}, y_{1}, y_{2}, \ldots\right)$ is nonnegative with respect to $[0, b]$. Then for any $\alpha_{1} \in(0,+\infty)$, there exists a linear operator $T \in L(X, Y)$ such that

$$
T\left(\varphi_{j}\right)=y_{j}, j \in \mathbb{N}, j \geq 1, T\left(\psi_{k}\right) \geq \alpha_{1} u_{0}, k \in \mathbb{N}, T(\varphi) \leq\left(2+\alpha_{1}\right)\|\varphi\| u_{0}, \quad \varphi \in X .
$$

If we additionally assume that $\alpha_{1} \geq 1$, and $Y$ is a Banach lattice whose closed unit ball is $\left[-u_{0}, u_{0}\right]$, then $\|T\| \leq 2+\alpha_{1}, T$ is continuous and positive.

Proof. One applies Theorem 2.4, where $S$ stands for $\operatorname{Span}\left\{\varphi_{j} ; j=1\right\}$ and $C$ stands for the convex hull of the set $\left\{\psi_{k} ; k \in \mathbb{N}\right\}$. We have $\|s-a\| \geq|s(0)-a(0)|=|0-1|=1$ for all $s \in S$ and all $a \in C$, so that $d(S, C)=1$. This can be written as $(S+B(0,1)) \cap C=\emptyset$, so that $V$ stands for $B(0,1)$ and $\|\cdot\|_{\infty}$ stands for $P_{V}$ in Theorem 2.4. On the other hand, $\left\|\psi_{k}\right\|=1$, $k \in \mathbb{N}$ implies that $\|a\| \leq 1$ for all $a \in C$, so that the number 1 stands for $\alpha$ in Theorem 2.4. We also take in Theorem 2.4 $\tilde{y}=\alpha_{1} u_{0}$. Define

$$
f\left(\sum_{j=1}^{n} \lambda_{j} \varphi_{j}\right)=\sum_{j=1}^{n} \lambda_{j} y_{j}, n \in \mathbb{N}, n \geq 1, \lambda_{j} \in \mathbb{R}, j=1, \ldots, n .
$$

Let $\sum_{j=1}^{n} \lambda_{j} \varphi_{j} \in S \cap B(0,1)$. Using the hypothesis on the sequence which is nonnegative on $[0, b]$, one has:

$$
\begin{gathered}
\sup \left\{\left|\sum_{j=1}^{n} \lambda_{j} t^{j}\right| ; t \in[0, b]\right\} \leq 1 \Longrightarrow \\
\sum_{j=1}^{n} \lambda_{j} t^{j}+1 \geq 0,1-\sum_{j=1}^{n} \lambda_{j} t^{j} \geq 0, \forall t \in[0, b] \Longrightarrow \\
\sum_{j=1}^{n} \lambda_{j} y_{j}+u_{0} \geq 0, u_{0}-\sum_{j=1}^{n} \lambda_{j} y_{j} \geq 0 \Longrightarrow \\
f\left(\sum_{j=1}^{n} \lambda_{j} \varphi_{j}\right)=\sum_{j=1}^{n} \lambda_{j} y_{j} \in\left[-u_{0}, u_{0}\right] \Longrightarrow \\
f(S \cap B(0,1)) \subseteq\left[-u_{0}, u_{0}\right] .
\end{gathered}
$$

The conclusion is that $f$ satisfies the conditions of Theorem 2.4. According to this theorem, there exists a linear extension $T$ of $f$ such that

$$
\left.T\right|_{C} \geq \alpha_{1} u_{0}, T(\varphi) \leq\left(1+1+\alpha_{1}\right)\|\varphi\| u_{0}=\left(2+\alpha_{1}\right)\|\varphi\| u_{0}, \varphi \in X
$$

Next, we prove the last assertion of the statement. For this, additionally assume that $\alpha_{1} \geq 1$ and $Y$ is a Banach lattice. From what is already proved, replacing $\varphi$ by $-\varphi$, we infer that $|T(\varphi)| \leq\left(2+\alpha_{1}\right)\|\varphi\| u_{0}$, which further yields:

$$
\|T(\varphi)\| \leq\left(2+\alpha_{1}\right)\|\varphi\|, \varphi \in X,
$$

since the norm on $Y$ is solid and $\left\|u_{0}\right\|=1$. Hence $T$ is continuous and $\|T\| \leq 2+\alpha_{1}$. Thanks to its continuity and to Weierstrass approximation theorem, to prove the positivity of $T$, it is sufficient to show that $T(p) \geq 0$ for all polynomials $p$, with $p(t) \geq 0$ for all $t \in[0, b]$. Indeed, let $p(t)$ be defined by $p(t)=\lambda_{0}+\sum_{j=1}^{n} \lambda_{j} t^{j} \geq 0, t \in[0, b]$. According to our hypothesis, it holds that

$$
\begin{gathered}
\lambda_{0} u_{0}+\sum_{j=1}^{n} \lambda_{j} y_{j} \geq 0 \Longrightarrow \sum_{j=1}^{n} \lambda_{j} y_{j} \geq-\lambda_{0} u_{0} \Longrightarrow \\
T\left(\lambda_{0} \mathbf{1}+\sum_{j=1}^{n} \lambda_{j} \varphi_{j}\right)=\lambda_{0} T\left(\psi_{0}\right)+\sum_{j=1}^{n} \lambda_{j} y_{j} \geq \lambda_{0} \alpha_{1} u_{0}-\lambda_{0} u_{0}=\lambda_{0}\left(\alpha_{1}-1\right) u_{0} \geq 0 .
\end{gathered}
$$

We have used the fact that $\lambda_{0}=p(0)=0$. The proof is now complete. 
The preceding result leads to the next one. We need the following basic example of a commutative algebra of self-adjoint operators, which is also an order complete vector lattice. Let $H$ be a Hilbert space and $A$ be a self-adjoint operator acting on $H$. Let $Y=Y(A)$ be the space of self-adjoint operators acting on $H$ (see pp.303-305 in [7]). Namely, if $\mathcal{A}=\mathcal{A}(H)$ is the space of all self-adjoint operators acting on $H$ then the natural order relation on $\mathcal{A}$ is, by definition,

$$
V \leq W \Longleftrightarrow\langle V h, h\rangle \leq\langle W h, h\rangle \forall h \in H
$$

With respect to this order relation, $\mathcal{A}(H)$ is an ordered Banach space, which is not a lattice. Moreover, the multiplication operation on $\mathcal{A}(H)$ is not commutative. Therefore, using the following notations, we define a suitable subspace $Y(A)$ of $\mathcal{A}(H)$ :

$$
\mathrm{Y}_{1}(\mathrm{~A})=\{\mathrm{V} \in \mathcal{A} ; \mathrm{AV}=\mathrm{VA}\}, \mathrm{Y}(\mathrm{A})=\left\{\mathrm{W} \in \mathrm{Y}_{1}(\mathrm{~A}) ; \mathrm{WV}=\mathrm{VW}, \forall \mathrm{V} \in \mathrm{Y}_{1}(\mathrm{~A})\right\}
$$

Then $Y(A)$ is the codomain space that we are interested in for the next two results. This space is an order complete Banach lattice (see [7]) and is a commutative real algebra (this last assertion is obvious).

Theorem 3.4 (see [22], Corollary 32). Let $H$ be a Hilbert space, $A \in \mathcal{A}(H)$ a positive self-adjoint operator acting on $H$, and $Y=Y(A)$ the order complete Banach lattice defined by (1). Take $b \in \mathbb{R}_{+}$such that the spectrum $\sigma(A)=[0, b]$ and $\alpha_{1} \geq 1$. Then there exists a nondecreasing mapping $\sigma:[0, b] \rightarrow Y$ such that

$$
\int_{0}^{b} t^{j} d \sigma=A^{j}, j \in \mathbb{N}, j \geq 1, \int_{0}^{b} e^{-k t} d \sigma \geq \alpha_{1} I, k \in \mathbb{N}, \int_{0}^{b} \varphi(t) d \sigma \leq\left(2+\alpha_{1}\right)\|\varphi\| I, \varphi \in C([0, b]) .
$$

In particular, for such a mapping $\sigma$, the following inequality holds

$$
\alpha_{1}+1 \leq\|\sigma(b)-\sigma(0)\|+\|\exp (-k U)\|, k \in \mathbb{N} .
$$

Proof. We apply Theorem 3.3 to

$$
Y=Y(A), u_{0}=I \in Y, y_{j}=A^{j}, j \in\{1,2, \ldots\}, \psi_{k}(t)=\exp (-k t), k \in \mathbb{N}, t \in[0, b], \varphi_{j}(t)=t^{j}, j \in \mathbb{N}, j \geq 1, t \in[0, b]
$$

Here, $I: H \rightarrow H$ is the identity operator. The sequence $\left(I, A, A^{2}, \ldots, A^{n}, \ldots\right)$ is nonnegative with respect to the interval $[0, b]$. Indeed, if $p(t)=\lambda_{0}+\lambda_{1} t+\cdots+\lambda_{n} t^{n} \geq 0 \forall t \in[0, b]=\sigma(A)$, then we obtain:

$$
\lambda_{0}+\lambda_{1} A+\cdots+\lambda_{n} A^{n}=\int_{\sigma(A)} p(t) d E_{A} \geq 0,
$$

since the spectral measure $d E_{A}$ is positive. Thus, all the requirements of the statement of Theorem 3.3 are satisfied. According to this theorem, there exists a linear positive (bounded) operator $T: C([0, b]) \rightarrow Y$, with the properties specified in the conclusion of Theorem 3.3. According to [7] (see p.272), there is a monotone increasing mapping $\sigma:[0, b] \rightarrow Y$ such that

$$
T(\varphi)=\int_{0}^{b} \varphi(t) d \sigma, \varphi \in C([0, b])
$$

This leads to:

$$
\begin{gathered}
\int_{0}^{b} t^{j} d \sigma=T\left(\varphi_{j}\right)=A^{j}, j \in \mathbb{N}, j \geq 1, \int_{0}^{b} \exp (-k t) d \sigma=T\left(\psi_{k}\right) \geq \alpha_{1} I, \quad k \in \mathbb{N} \\
\int_{0}^{b} \varphi(t) d \sigma=T(\varphi) \leq\left(2+\alpha_{1}\right)\|\varphi\| I, \varphi \in C([0, b]) .
\end{gathered}
$$

Next, we prove the last inequality of the statement. From the previously mentioned inequality, for any $k \in \mathbb{N}$ we have

$$
\begin{gathered}
\alpha_{1} I \leq \int_{0}^{b} e^{-k t} d \sigma=\int_{0}^{b}\left(1+\sum_{m=1}^{\infty} \frac{(-k)^{m}}{m !} t^{m}\right) d \sigma=\sigma(b)-\sigma(0)+\sum_{m=1}^{\infty} \frac{(-k)^{m}}{m !} A^{m}=\sigma(b)-\sigma(0)-I+\exp (-k A) \Longrightarrow \\
\left(\alpha_{1}+1\right) I \leq \sigma(b)-\sigma(0)+\exp (-k A) .
\end{gathered}
$$

Due to the fact that the norm on the Banach lattice $Y$ is monotone (increasing) on the positive cone $Y_{+}$, the preceding inequality implies

$$
\alpha_{1}+1 \leq\|\sigma(b)-\sigma(0)\|+\|\exp (-k A)\|, k \in \mathbb{N} .
$$

The proof is now complete. 
In the particular case when $H=\mathbb{R}^{n}, n \geq 2$, and $A$ is a symmetric $n \times n$ matrix with real entries, the space $Y(A)$ defined by (1) is isomorphic to a commutative algebra of the space $\operatorname{Sym}(n, \mathbb{R})$ of all symmetric $n \times n$ matrices with real entries, and is also an order complete Banach lattice with respect to the trace of the usual order relation defined in $\operatorname{Sym}(n, \mathbb{R})$ on $Y(A)$.

We go on with a Mazur-Orlicz type result in concrete spaces. Let $H$ be an arbitrary Hilbert space, $A \in \mathcal{A}(H)$ be a positive self-adjoint operator, $Y=Y(A)$ be the space defined by (1). Let $X$ be the space $C(\sigma(A)), \varphi_{j}(t)=t^{j}, j \in \mathbb{N}, t \in \sigma(A)$, and $\left(B_{j}\right)_{j \in \mathbb{N}}$ be a sequence in $Y$.

Theorem 3.5. The following statements are equivalent:

(a). There exists a linear bounded positive operator $T \in B_{+}(X, Y)$ such that

$$
T\left(\varphi_{j}\right) \geq B_{j}, j \in \mathbb{N}, T(\varphi) \leq \int_{\sigma(U)}|\varphi| d E_{U}, \forall \varphi \in X,\|T\| \leq 1
$$

(b). $B_{j} \leq A^{j}, j \in \mathbb{N}$.

Proof. The implication $(\mathrm{a}) \Rightarrow(\mathrm{b})$ is obvious:

$$
B_{j} \leq T\left(\varphi_{j}\right) \leq \int_{\sigma(A)}\left|\varphi_{j}\right| d E_{A}=\int_{\sigma(U)} t^{j} d E_{A}=A^{j}, j \in \mathbb{N}
$$

we have used the positivity of the operator $A$ which leads to $\left|\varphi_{j}\right|=\varphi_{j}$ on $\sigma(A) \subset \mathbb{R}_{+}$. For the converse, that is (b) $\Rightarrow$ (a), one applies Theorem 2.5, where $J$ stands for $\mathbb{N}, x_{j}$ stands for $\varphi_{j}$ and $y_{j}$ stands for $B_{j}, j \in \mathbb{N}$. Let $J_{0}$ and $\left\{\lambda_{j}\right\}_{j \in J_{0}}$ be as mentioned in part (b) of Theorem 2.5. The following implications hold

$$
\sum_{j \in J_{0}} \lambda_{j} \varphi_{j} \leq \varphi \in X \Rightarrow \sum_{j \in J_{0}} \lambda_{j} \int_{\sigma(A)} \varphi_{j} d E_{A}=\sum_{j \in J_{0}} \lambda_{j} A^{j} \leq \int_{\sigma(A)} \varphi d E_{A} \leq \int_{\sigma(A)}|\varphi| d E_{A}:=P(\varphi) .
$$

The positivity of the spectral measure $d E_{A}$ has been used. On the other hand, the hypothesis (b), the fact that the scalars $\lambda_{j}$ are nonnegative and the preceding evaluation yield

$$
\lambda_{j} B_{j} \leq \lambda_{j} A^{j} \quad \forall j \Rightarrow \sum_{j \in J_{0}} \lambda_{j} B_{j} \leq \sum_{j \in J_{0}} \lambda_{j} A^{j} \leq P(\varphi),
$$

where $P(\varphi)$ is defined above. Thus, the implication (b) of Theorem 2.5 is satisfied. Application of the latter theorem leads to the existence of a "feasible solution" $T$ having the property mentioned in part (a) of the present theorem. The last property is a consequence of the preceding one, using the fact that the norm on $Y$ is solid. Namely,

$$
\begin{gathered}
\pm T(\varphi)=T( \pm \varphi) \leq \int_{\sigma(A)}|\varphi| d E_{A}:=P(\varphi) \Longrightarrow \\
|T(\varphi)| \leq P(\varphi) \Longrightarrow\|T(\varphi)\| \leq\|P(\varphi)\| \leq\|\varphi\| \cdot\|I\|=\|\varphi\|, \varphi \in X .
\end{gathered}
$$

Hence $\|T\| \leq 1$. This concludes the proof.

For the Markov moment problem discussed in Theorem 3.2, we recall one of its applications (see [2] and [23]). A convex subset $\mathcal{C}$ of the real vector space $X$ is called finite-simplicial if for any finite dimensional compact $K \subseteq \mathcal{C}$, there is a finite dimensional simplex $\mathcal{S}$ such that $K \subseteq \mathcal{S} \subseteq \mathcal{C}$. Information about finite-dimensional simplexes can be found in [18].

Theorem 3.6 (see [2], Corollary 3.5). Let $X$ be a vector space, $\mathcal{C} \subseteq X$ be a finite-simplicial set, $f: \mathcal{C} \rightarrow \mathbb{R}$ be a convex function, $g: \mathcal{C} \rightarrow \mathbb{R}$ be a concave function, such that $f \leq g$ on $\mathcal{C}$. There exists an affine function $h: \mathcal{C} \rightarrow \mathbb{R}$, satisfying the sandwich condition $f \leq h \leq g$ on $\mathcal{C}$.

In Theorem 3.6, the novelty is that a finite-simplicial set $\mathcal{C} \subseteq X$ may be unbounded in any locally convex topology on $\mathcal{C}$. Next, we review the statement of a topological version of the preceding theorem.

Theorem 3.7 (see [23], Theorem 4). Let X be an ordered Banach space. Assume that the positive cone $X_{+}$is finite-simplicial and there exists $x_{0} \in X_{+}$such that $X_{+}-x_{0}$ contains a balanced and absorbing convex subset. Let

$$
f,-g: X_{+} \rightarrow \mathbb{R}
$$

be convex continuous functions such that $f \leq g$. Assume also that $f(\mathbf{0})=g(\mathbf{0})=0$. Then there exists a continuous linear functional $L: X \rightarrow \mathbb{R}$ such that $f \leq L \leq$ on $X_{+}$. 


\section{Polynomial approximation on unbounded subsets and the classical Markov moment problem}

We recall the classical formulation of the moment problem, under the terms of Stieltjes (see the basic book of Akhiezer [1] for details): find the repartition of the positive mass on the nonnegative semi-axis if the moments of arbitrary orders $j$ $(j=0,1,2, \ldots)$ are given. More precisely, in the Stieltjes moment problem, a sequence of real numbers $\left(y_{j}\right)_{j \geq 0}$ is given and one looks for a nondecreasing real function $\sigma(t)(t \geq 0)$, which satisfies the moment conditions:

$$
\int_{0}^{\infty} t^{j} d \sigma=y_{j}, \quad(j=0,1,2, \ldots)
$$

This is a one-dimensional moment problem on an unbounded interval. Namely, it is an interpolation problem with the constraint on the positivity of the measure $d \sigma$. The numbers $y_{j}, j \in \mathbb{N}=\{0,1,2, \ldots\}$ are called the moments of the measure $d \sigma$. The present section concerns firstly the existence problem. However, the uniqueness is studied as well. The moment problem is an inverse problem: we are looking for an unknown measure, starting from its moments. The direct problem might be: being given the measure $d \sigma$, compute its moments $\int_{0}^{\infty} t^{j} d \sigma, j=0,1,2, \ldots$. The connection with the positive polynomials and extensions of linear positive functional and operators is quite clear. Namely, if one takes $\varphi_{j}(t):=t^{j}$, $j \in \mathbb{N}, t \in[0,+\infty), \mathcal{P}$ the vector space of polynomials with real coefficients and

$$
T_{0}: \mathcal{P} \rightarrow \mathbb{R}, T_{0}\left(\sum_{j \in J_{0}} \alpha_{j} \varphi_{j}\right):=\sum_{j \in J_{0}} \alpha_{j} y_{j},
$$

where $J_{0} \subset \mathbb{N}$ is a finite subset, then the moment conditions $T_{0}\left(\varphi_{j}\right)=y_{j}, j \in \mathbb{N}$ are clearly verified. It remains to check whether the linear form $T_{0}$ has nonnegative values at all nonnegative polynomials. If this condition is satisfied, due to Haviland theorem, there exists a positive regular Borel measure $\mu$ on $[0,+\infty)$ such that $\int_{0}^{+\infty} t^{j} d \mu=y_{j}, j \in \mathbb{N}$. In other words, the measure $\mu$ satisfies the condition

$$
\int_{0}^{+\infty} p d \mu=T_{0}(p), p \in \mathcal{P}
$$

The multidimensional moment problem is formulated similarly. Let $n \in \mathbb{N}, n \geq 2$, and $F \subseteq \mathbb{R}^{n}$ be a closed subset. One denotes

$$
\varphi_{j}(t)=t^{j}=t_{1}^{j_{1}} \cdots t_{n}^{j_{n}}, j=\left(j_{1}, \ldots, j_{n}\right) \in \mathbb{N}^{n}, t=\left(t_{1}, \ldots, t_{n}\right) \in F, \mathbb{R}_{+}=[0,+\infty), \mathcal{P}_{+}(F)=\{p \in \mathcal{P} ; p(t) \geq 0 \forall t \in F\} .
$$

Being given a sequence $\left(y_{j}\right)_{j \in \mathbb{N}^{n}}$ of real numbers, the existence moment problem means to find a necessary and sufficient condition on the numbers $y_{j}, j \in \mathbb{N}$ for the existence of a positive regular Borel measure $\mu$ on $F$ such that

$$
\int_{F} t^{j} d \mu=y_{j}, j \in \mathbb{N}^{n}
$$

Due to Haviland theorem [11] (which works in more than one dimension), the desired condition is

$$
T_{0}(p) \geq 0 \text { for all } p \in \mathcal{P}_{+}(F),
$$

where $T_{0}$ is defined by (2) and $j \in \mathbb{N}^{n}$. It is well-known that there exists nonnegative polynomials on the entire space $\mathbb{R}^{n}, n \geq 2$, which are not sums of squares of polynomials (see [3,31]), unlike the case $n=1$, where $p \in \mathcal{P}_{+}(\mathbb{R}) \Longleftrightarrow p=p_{1}^{2}+p_{2}^{2}$ for some $p_{1}, p_{2} \in \mathbb{R}[t]=\mathcal{P}$. Also, for $p \in \mathcal{P}$, we have $p(t) \geq 0$ for all $t \in[0,+\infty)$ if and only if $p(t)=p_{1}^{2}(t)+t p_{2}^{2}(t) \forall t \in[0,+\infty)$ for some $p_{1}, p_{2} \in \mathcal{P}$. These well-known facts make possible writing condition (4) for $F=\mathbb{R}$ and for $F=\mathbb{R}_{+}$in terms of quadratic forms. For $F=\mathbb{R}^{n}$, or $F=\mathbb{R}_{+}^{n}, n \geq 2$, this is no more possible. If there exists a measure $\mu$ such that (3) holds, we say that $\left(y_{j}\right)_{j \in \mathbb{N}^{n}}$ is a sequence of moments or a moment sequence, and $\mu$ is a representing measure for $\left(y_{j}\right)_{j \in \mathbb{N}^{n}}$. From now on, by a measure on $F$ we mean a positive regular measure on $F$ with finite moments of all orders. A sequence $\left(y_{j}\right)_{j \in \mathbb{N}^{n}}$ of numbers is called positive semi-definite if for any finite subset $J_{0} \subset \mathbb{N}^{n}$ and for any $\left\{\lambda_{j} ; j \in J_{0}\right\} \subset \mathbb{R}$, we have

$$
\sum_{i, j \in J_{0}} \lambda_{i} \lambda_{j} y_{i+j} \geq 0
$$

Clearly, any moment sequence is positive semi-definite. Indeed, if $\left(y_{j}\right)_{j \in \mathbb{N}^{n}}$ is a moment sequence, we can write

$$
\sum_{i, j \in J_{0}} \lambda_{i} \lambda_{j} y_{i+j}=\sum_{i, j \in J_{0}} \lambda_{i} \lambda_{j} \int_{F} t^{i+j} d \mu=\int_{F}\left(\sum_{j \in J_{0}} \lambda_{j} t^{j}\right)^{2} d \mu \geq 0
$$


For $n=1$, the converse is true since any non-negative polynomial on $\mathbb{R}$ is a sum of (two) squares of polynomials and a square of a polynomial can be written as

$$
\left(\sum_{j \in J_{0}} \lambda_{j} \varphi_{j}\right)^{2}=\sum_{i, j \in J_{0}} \lambda_{i} \lambda_{j} \varphi_{i+j}
$$

then one applies Haviland theorem. For $n \geq 2$, there exist positive semi-definite sequences that are not moment sequences (see [4]). This is one of the main differences between the one-dimensional and multidimensional moment problems. More difficulties appear when one studies the uniqueness of the solution. The present section solves a part of these problems by means of polynomial approximation on Cartesian products of closed unbounded intervals. If in (3), the $y_{j}, j \in \mathbb{N}^{n}$, are elements of an ordered vector space $Y$ then the solution is a positive linear operator $T: X \rightarrow Y$, where $X$ is usually ordered Banach space (or even a Banach lattice) of real functions on $F$, which contains $\mathcal{P}$ and the space $C_{0}(F)$ of all continuous compactly supported real functions defined on $F$. For example, if $\nu$ is a positive regular Borel measure on $F$ with finite moments of all orders then we can take $X=L_{\nu}^{1}(F)$. Additionally, assuming that $\nu$ is a moment determinate measure, we recall in Lemma 4.1 that $\mathcal{P}_{+}(F)$ is dense in $\left(L_{\nu}^{1}(F)\right)_{+}$. In particular, $\mathcal{P}$ is dense in $X=L_{\nu}^{1}(F)$. If $Y$ is an order complete Banach lattice and $T: X=L_{\nu}^{1}(F) \rightarrow Y$ is linear and positive, satisfying the interpolation moment conditions

$$
T\left(\varphi_{j}\right)=y_{j}, j \in \mathbb{N}^{n},
$$

then $T$ is called a solution of the the moment problem defined by (5). The order completeness of $Y$ is necessary for extending $T_{0}$ from a suitable chosen subspace of $X$ to the entire space $X$, preserving positivity property. Then the solution $T$ is unique, since any positive linear operator acting between Banach lattices is continuous (this is also true for $T$ acting on ordered Banach spaces (see [17])), and $\mathcal{P}$ is dense in $X$. We recall that, by definition, the measure $\nu$ is moment determinate ( $M$-determinate) if it is uniquely determinate by its moments $\int_{F} t^{j} d \nu, j \in \mathbb{N}^{n}$. In what follows, we prove and apply polynomial approximation on unbounded subsets in order to characterize as simple as possible the existence and uniqueness of the solution $T: L_{\nu}^{1}(F) \rightarrow Y$ which satisfies the moment conditions (5), is positive (linear) and is dominated by a continuous convex operator $P: X \rightarrow Y$ on $X=L_{\nu}^{1}(F)$. In most cases, $P$ is a continuous sublinear operator from $X$ to $Y$. Another interesting sandwich condition on the solution $T$ is $T_{1} \leq T \leq T_{2}$ on $X_{+}$, where $T_{1} \leq T_{2}$ on $X_{+}$. Here $T_{1}$ and $T_{2}$ are given bounded linear operators from $X$ to $Y$. In this framework, we can control the norm of the solution $T$ as well. We start by a key approximation lemma.

Lemma 4.1 (see [24], Lemma 3). Let $F \subseteq \mathbb{R}^{n}$ be an unbounded closed subset and let $\nu$ be an M-determinate measure on $F$ (with finite moments of all natural orders). Then, for any $x \in C_{c}(F), x(t) \geq 0, \forall t \in F$, there exists a sequence $\left(p_{m}\right)_{m}, p_{m} \geq x, m \in \mathbb{N}, p_{m} \rightarrow x$ in $L_{\nu}^{1}(F)$. In particular, it holds that

$$
\lim _{m} \int_{F} p_{m}(t) d \nu=\int_{F} x(t) d \nu
$$

the cone $\mathcal{P}_{+}$of nonnegative polynomials is dense in $\left(L_{\nu}^{1}(F)\right)_{+}$, and $\mathcal{P}$ is dense in $L_{\nu}^{1}(F)$.

As an application of Lemma 4.1, we derive the next result.

Theorem 4.1 (see [24], Theorem 2). Let $F$ be a closed unbounded subset of $\mathbb{R}^{n}, Y$ be an order complete Banach lattice, $\left(y_{j}\right)_{j \in \mathbb{N}^{n}}$ be a given sequence in $Y, \nu$ be a positive regular $M$-determinate Borel measure on $F$ with finite moments of all orders. Let $T_{2} \in B\left(L_{\nu}^{1}(F), Y\right)$ be a linear positive bounded operator from $L_{\nu}^{1}(F)$ to $Y$. The following statements are equivalent

(a). There exists a unique linear operator $\tilde{T} \in B\left(L_{\nu}^{1}(F), Y\right)$ such that $\tilde{T}\left(\varphi_{j}\right)=y_{j}, j \in \mathbb{N}^{n}, \tilde{T}$ is between 0 and $T_{2}$ on the positive cone of $L_{\nu}^{1}(A)$, and $\|\tilde{T}\| \leq\left\|T_{2}\right\|$.

(b). For any finite subset $J_{0} \subset \mathbb{N}^{n}$, and for any $\left\{a_{j}\right\}_{j \in J_{0}} \subset \mathbb{R}$, we have

$$
\sum_{j \in J_{0}} a_{j} \varphi_{j} \geq 0 \text { on } F \Longrightarrow 0 \leq \sum_{j \in J_{0}} a_{j} y_{j} \leq \sum_{j \in J_{0}} a_{j} T_{2}\left(\varphi_{j}\right)
$$

Lemma 4.2. If $\nu=\nu_{1} \times \cdots \times \nu_{n}$ is a product of $n M$-determinate measures on $\mathbb{R}$, then any non-negative continuous compactly supported function $f$ in $X=L_{\nu}^{1}\left(\mathbb{R}^{n}\right)$ can be approximated with sums of products

$$
p_{1} \otimes \cdots \otimes p_{n},\left(p_{1} \otimes \cdots \otimes p_{n}\right)\left(t_{1}, \ldots, t_{n}\right):=p_{1}\left(t_{1}\right) \cdots p_{n}\left(t_{n}\right),
$$

where $p_{j}$ are nonnegative polynomial on the entire real line, $j=1, \ldots, n$, such that any such sum of special polynomials dominates $f$ on $\mathbb{R}^{n}$. 
To prove Lemma 4.2, one can use approximating Bernstein polynomial of $n$ variables. Then, on applies Lemma 4.1 in each separate variable, for $n=1, F=\mathbb{R}$, and the Fubini theorem.

The next result follows from Lemma 4.2, also using the explicit form of nonnegative polynomials on the entire real axis as a sum of (two) squares of polynomials with real coefficients.

Corollary 4.1 (see [24], Theorem 6). Let $X=L_{\nu}^{1}\left(\mathbb{R}^{n}\right)$, where $\nu=\nu_{1} \times \cdots \times \nu_{n}$ is a product of $n$ M-determinate positive regular Borel measure on $\mathbb{R}$ with finite moments of all orders, $Y$ be an order complete Banach lattice, $\left(y_{j}\right)_{j \in \mathbb{N}_{n}}$ be a sequence in $Y$, and $T_{2} \in B_{+}(X, Y)$. The following statements are equivalent:

(a). There exists a unique (bounded) linear operator $T \in B(X, Y)$ such that $T\left(\varphi_{j}\right)=y_{j}, j \in \mathbb{N}^{n}$, $T$ is between zero and $T_{2}$ on the positive cone of $X,\|T\| \leq\left\|T_{2}\right\|$;

(b). For any finite subset $J_{0} \subset \mathbb{N}^{n}$ and for any $\left\{\lambda_{j} ; j \in J_{0}\right\} \subset \mathbb{R}$, one has

$$
\sum_{j \in J_{0}} \lambda_{j} \varphi_{j}(t) \geq 0 \forall t \in \mathbb{R}^{n} \Longrightarrow \sum_{j \in j_{0}} \lambda_{j} y_{j} \in Y_{+},
$$

and for any finite subsets $J_{k} \subset \mathbb{N}, k=1, \ldots, n$, and for any $\left\{\lambda_{j_{k}}\right\}_{j_{k} \in J_{k}} \subset \mathbb{R}, k=1, \ldots, n$, the following inequality holds

$$
\begin{aligned}
& \sum_{i_{1}, j_{1} \in J_{1}}\left(\ldots\left(\sum_{i_{n}, j_{n} \in J_{n}} \lambda_{i_{1}} \lambda_{j_{1}} \ldots \lambda_{i_{n}} \lambda_{j_{n}} y_{i_{1}+j_{1}, \ldots, i_{n}+j_{n}}\right) \ldots\right) \leq \\
& \sum_{i_{1}, j_{1} \in J_{1}}\left(\ldots\left(\sum_{i_{n}, j_{n} \in J_{n}} \lambda_{i_{1}} \lambda_{j_{1}} \ldots \lambda_{i_{n}} \lambda_{j_{n}} T_{2}\left(\varphi_{i_{1}+j_{1}, \ldots, i_{n}+j_{n}}\right) \ldots\right) .\right.
\end{aligned}
$$

Theorem 4.2 (see [24], Theorem 7). Let $\nu$ be as in Lemma 4.2, $X=L_{\nu}^{1}\left(\mathbb{R}^{n}\right)$, and $Y$ be a Banach lattice. Assume that $T$ is a linear bounded operator from $X$ to $Y$. The following statements are equivalent:

(a). $T$ is positive on the positive cone of $X$.

(b). For any finite subsets $J_{k} \subset \mathbb{N}, k=1, \ldots, n$, and for any $\left\{\lambda_{j_{k}}\right\}_{j_{k} \in J_{k}} \subset \mathbb{R}, k=1, \ldots, n$, the following inequality holds

$$
0 \leq \sum_{i_{1}, j_{1} \in J_{1}}\left(\cdots\left(\sum_{i_{n}, j_{n} \in J_{n}} \lambda_{i_{1}} \lambda_{j_{1}} \cdots \lambda_{i_{n}} \lambda_{j_{n}} T\left(x_{i_{1}+j_{1}}, \cdots, i_{n}+j_{n}\right)\right) \cdots\right) .
$$

Proof. Note that (b) says that $T$ is positive on the convex cone generated by special positive polynomials $p_{1} \otimes \cdots \otimes p_{n}$, each factor of any term in the sum being non-negative on the whole real axis. Consequently, (a) $\Rightarrow(b)$ is clear. In order to prove the converse, observe that any non-negative element of $X$ can be approximated by non-negative continuous compactly supported functions. Such functions can be approximated by the sums of tensor products of positive polynomials in each separate variable, the latter being sums of squares. The conclusion is that any nonnegative function from $X$ can be approximated in $X=L_{\nu}^{1}\left(\mathbb{R}^{n}\right)$ by the sums of tensor products of squares of polynomials in each separate variable. We know that on such special polynomials, $T$ admits values in $Y_{+}$, according to the condition (b). Now, the desired conclusion is a consequence of the continuity of $T$, also using the fact that the positive cone of $Y$ is closed. This concludes the proof.

Lemma 4.3. If $\nu=\nu_{1} \times \cdots \times \nu_{n}$ is a product of $n$ M-determinate positive regular Borel measures on $\mathbb{R}_{+}=[0,+\infty)$ with finite moments of all natural orders then any nonnegative continuous compactly supported function $f$ in $X=L_{\nu}^{1}\left(\mathbb{R}_{+}^{n}\right)$ can be approximated by means of sums of tensor products $p_{1} \otimes \cdots \otimes p_{n}$, where $p_{j}$ is a nonnegative polynomial on the real nonnegative semi-axis, $j=1, \ldots, n$, such that all these sums of special nonnegative polynomials dominate $f$ on $\mathbb{R}_{+}^{n}$.

Corollary 4.2 (see [24], Theorem 5). Let $X$ be as in Lemma 4.3, $\left(y_{j}\right)_{j \in \mathbb{N}^{n}}$ be a sequence in $Y$, where $Y$ is an order complete Banach lattice. Let $T_{2} \in B_{+}(X, Y)$ be a positive (bounded) linear operator. The following statements are equivalent:

(a). There exists a unique (bounded) linear operator $T \in B(X, Y)$ such that $T\left(\varphi_{j}\right)=y_{j}, j \in \mathbb{N}^{n}, T$ is between zero and $T_{2}$ on the positive cone of $X,\|T\| \leq\left\|T_{2}\right\|$.

(b). For any finite subset $J_{0} \subset \mathbb{N}^{n}$ and for any $\left\{\lambda_{j} ; j \in J_{0}\right\} \subset \mathbb{R}$, one has

$$
\sum_{j \in J_{0}} \lambda_{j} \varphi_{j}(t) \geq 0 \forall t \in \mathbb{R}_{+}^{n} \Longrightarrow \sum_{j \in j_{0}} \lambda_{j} y_{j} \in Y_{+}
$$


and for any finite subsets $J_{k} \subset \mathbb{N}, k=1, \ldots, n$, and for any $\left\{\lambda_{j_{k}}\right\}_{j_{k} \in J_{k}} \subset \mathbb{R}, k=1, \ldots, n$, the following inequality holds

$$
\begin{gathered}
\sum_{i_{1}, j_{1} \in J_{1}}\left(\ldots\left(\sum_{i_{n}, j_{n} \in J_{n}} \lambda_{i_{1}} \lambda_{j_{1}} \ldots \lambda_{i_{n}} \lambda_{j_{n}} y_{i_{1}+j_{1}+l_{1}, \ldots, i_{n}+j_{n}+l_{n}}\right) \ldots\right) \leq \\
\sum_{i_{1}, j_{1} \in J_{1}}\left(\ldots\left(\sum_{i_{n}, j_{n} \in J_{n}} \lambda_{i_{1}} \lambda_{j_{1}} \ldots \lambda_{i_{n}} \lambda_{j_{n}} T_{2}\left(\varphi_{i_{1}+j_{1}+l_{1}, \ldots, i_{n}+j_{n}+l_{n}}\right) \ldots\right),\left(l_{1}, \ldots, l_{n}\right) \in\{0,1\}^{n}\right.
\end{gathered}
$$

Next, we proceed with a stronger sandwich condition on the solution $T$.

Theorem 4.3 (see [26], Theorem 4). Let $F \subseteq \mathbb{R}^{n}$ be a closed unbounded subset, $\nu$ be a positive Borel moment determinate measure on $F$ having finite moments of all orders, $X=L_{\nu}^{1}(F), \varphi_{j}(t)=t^{j}, t \in F, j \in \mathbb{N}^{n}$. Let $Y$ be an order complete Banach lattice, $\left(y_{j}\right)_{j \in \mathbb{N}^{n}}$ be a given sequence of elements in $Y, T_{1}$ and $T_{2}$ be two bounded linear operators from $X$ to $Y$. Assume that there exists a sub-cone $\mathcal{P}_{++} \subseteq \mathcal{P}_{+}$such that each $f \in\left(C_{c}(F)\right)_{+}$can be approximated in $X$ by a sequence $\left(p_{l}\right)_{l}$, $p_{l} \in \mathcal{P}_{++}, p_{l} \geq f$ for all $l$. The following statements are equivalent:

(a). There exists a unique (bounded) linear operator $T: X \rightarrow Y, T\left(\varphi_{j}\right)=y_{j}, j \in \mathbb{N}^{n}, \mathbf{0} \leq T_{1} \leq T \leq T_{2}$ on $X_{+}$,

$$
\left\|T_{1}\right\| \leq\|T\| \leq\left\|T_{2}\right\|
$$

(b). For any finite subset $J_{0} \subset \mathbb{N}^{n}$ and for any $\left\{\alpha_{j} ; j \in J_{0}\right\} \subset \mathbb{R}$, the following implications hold

$$
\begin{gathered}
\sum_{j \in J_{0}} \alpha_{j} \varphi_{j} \in \mathcal{P}_{+} \Longrightarrow \sum_{j \in J_{0}} \alpha_{j} T_{1}\left(\varphi_{j}\right) \leq \sum_{j \in J_{0}} \alpha_{j} y_{j} \\
\sum_{j \in J_{0}} \alpha_{j} \varphi_{j} \in \mathcal{P}_{++} \Longrightarrow \sum_{j \in J_{0}} \alpha_{j} T_{1}\left(\varphi_{j}\right) \geq 0, \sum_{j \in J_{0}} \alpha_{j} y_{j} \leq \sum_{j \in J_{0}} \alpha_{j} T_{2}\left(\varphi_{j}\right) .
\end{gathered}
$$

Proof. We start by observing that the first condition (7) implies the positivity of the bounded linear operator $T_{1}$, via its continuity. Indeed, if $f \in\left(C_{c}(F)\right)_{+}, p_{l} \in \mathcal{P}_{++}, p_{l} \geq f$ for all $l, p_{l} \rightarrow f$ in $L_{\nu}^{1}(F)$, then, according to the first condition (7), $T_{1}\left(p_{l}\right) \geq 0$ for all $l \in \mathbb{N}$ and the continuity of $T_{1}$ yields

$$
T_{1}(f)=\lim _{l} T_{1}\left(p_{l}\right) \geq 0 .
$$

Since $\left(C_{c}(F)\right)_{+}$is dense in $X_{+}$via measure theory reasons, the continuity of $T_{1}$ implies $T_{1} \geq \mathbf{0}$ on $X_{+}$. Thus, $T_{1}$ is a positive linear operator. Next, we define $T_{0}: \mathcal{P} \rightarrow Y, T_{0}\left(\sum_{j \in J_{0}} \lambda_{j} \varphi_{j}\right)=\sum_{j \in J_{0}} \lambda_{j} y_{j}$, where the sums are finite and the coefficients $\lambda_{j}$ are arbitrary real numbers. Condition (6) says that $T_{0}-T_{1} \geq \mathbf{0}$ on $\mathcal{P}_{+}$. If we consider the vector subspace $X_{1}$ of $X$ formed by all functions $\psi \in X$ having the modulus $|\psi|$ dominated by a polynomial $p \in \mathcal{P}_{+}$on the entire set $F$, then $\mathcal{P}$ is a majorizing subspace of $X_{1}$ and $T_{0}-T_{1}$ is a positive linear operator on $\mathcal{P}$. Application of Theorem 2.1 leads to the existence of a positive linear extension $U: X_{1} \rightarrow Y$, of $T_{0}-T_{1}$. Obviously, $X_{1}$ contains $C_{c}(F)+\mathcal{P}:\left(p \in \mathcal{P} \Longrightarrow|p|=\sqrt{\mathbf{1} \cdot p^{2}}=\left(\mathbf{1}+p^{2}\right) / 2 \in \mathcal{P}\right)$. Indeed, since $\varphi \in C(F) \Longrightarrow|\varphi| \in\left(C_{0}(F)\right)_{+} \Longrightarrow|\varphi| \leq b 1 \in \mathcal{P}$ (according to Weierstrass' Theorem), we infer that $\varphi \in X_{1}$; here $b$ is a real number. Hence, $C_{0}(F) \subset X_{1}$. Now, let $p \in \mathcal{P}$; we observe that

$$
1+p^{2}-2|p|=(1-|p|)^{2} \geq 0
$$

which implies that

$$
|p|=\frac{1+p^{2}}{2} \in \mathcal{P} .
$$

By using the definition of $X_{1}$, one has $\mathcal{P} \subset X_{1}$. Consequently, $C_{c}(F)+\mathcal{P} \subset X_{1}$. Going back to the positive linear extension $U: X_{1} \rightarrow Y$ of $T_{0}-T_{1}$, we conclude that $\hat{T}_{0}=U+T_{1}: X_{1} \rightarrow Y$ is an extension of $T_{0}, \hat{T}_{0} \geq T_{1}$ on $\left(X_{1}\right)_{+}$, and $\hat{T}_{0}(p)=$ $T_{0}(p) \leq T_{2}(p)$ for all $p \in \mathcal{P}_{++}$, according to the last requirement (7). Our first conclusion is:

$$
T_{1}(p) \leq \hat{T}_{0}(p) \leq T_{2}(p) \text { for all } p \in \mathcal{P}_{++}, \hat{T}_{0}(\psi) \geq T_{1}(\psi) \geq 0, \quad \psi \in\left(X_{1}\right)_{+} .
$$

Our next goal is to prove the continuity of $\hat{T}_{0}$ on $C_{c}(F)$. Let $\left(f_{l}\right)_{l \geq 0}$ be a sequence of nonnegative continuous compactly supported functions, such that $f_{l} \rightarrow \mathbf{0}$ in $X_{1}$, and a sequence of polynomials $p_{l} \geq f_{l} \geq \mathbf{0}, p_{l} \in \mathcal{P}_{++}$for all $l$, such that the following convergence result holds: $\left\|p_{l}-f_{l}\right\|_{1} \rightarrow 0, l \rightarrow \infty$. Consequently,

$$
\left\|p_{l}\right\|_{1} \leq\left\|p_{l}-f_{l}\right\|_{1}+\left\|f_{l}\right\|_{1} \rightarrow 0, l \rightarrow \infty
$$


Now, (8) and the continuity of $T_{1}$ and $T_{2}$ yield:

$$
0 \leftarrow T_{1}\left(p_{l}\right) \leq \hat{T}_{0}\left(p_{l}\right) \leq T_{2}\left(p_{l}\right) \rightarrow 0,
$$

hence $\hat{T}_{0}\left(p_{l}\right) \rightarrow 0$. It results:

$$
0 \leq T_{1}\left(f_{l}\right) \leq \hat{T}_{0}\left(f_{l}\right) \leq \hat{T}_{0}\left(p_{l}\right) \rightarrow 0,
$$

hence $\hat{T}_{0}\left(f_{l}\right) \rightarrow 0$. If $\left(g_{n}\right)_{n \geq 0}$ is an arbitrary sequence of compactly supported and continuous functions such that $g_{n} \rightarrow \mathbf{0}$ in $X_{1}$, then $g_{n}^{+} \rightarrow \mathbf{0}, g_{n}^{-} \rightarrow \mathbf{0}$. According to what we already have proved, we can write $\hat{T}_{0}\left(g_{n}^{+}\right) \rightarrow \mathbf{0}$ and $\hat{T}_{0}\left(g_{n}^{-}\right) \rightarrow 0$, which further yield $\hat{T}_{0}\left(g_{n}\right) \rightarrow 0$. This proves the continuity of $\hat{T}_{0}$ on $C_{c}(F)$ and the subspace $C_{c}(F)$ is dense in $X$. Moreover, $\left(C_{c}(F)\right)_{+}$is dense in $X_{+}$. Hence, there exists a unique continuous linear extension $T \in B(X, Y)$ of $\hat{T}_{0}$. For $f \in\left(C_{c}(F)\right)_{+}$, passing to the limit in (8) when $l \rightarrow \infty, p_{l} \rightarrow f, p_{l} \in \mathcal{P}_{++}$for all $l$ and using the continuity of the three operators $T_{1}, T, T_{2}$, it results $0 \leq T_{1}(f) \leq T(f) \leq T_{2}(f)$. Next, we apply the density of $\left(C_{c}(F)\right)_{+}$in $X_{+}$(see [29]), which yields

$$
\mathbf{0} \leq T_{1} \leq T \leq T_{2} \text { on } X_{+},\left\|T_{1}\right\| \leq\|T\| \leq\left\|T_{2}\right\|, T\left(\varphi_{j}\right)=T_{0}\left(\varphi_{j}\right)=y_{j}, j \rightarrow \mathbb{N}^{n} .
$$

For an arbitrary $\phi \in X$, the following inequalities hold via the preceding remarks:

$$
\pm T(\phi)=T( \pm \phi) \leq T(|\phi|) \leq T_{2}(|\phi|) \Longrightarrow|T(\phi)| \leq T_{2}(|\phi|) \Longrightarrow\|T(\phi)\| \leq\left\|T_{2}(|\phi|)\right\| \leq\left\|T_{2}\right\|\|\phi\| .
$$

Therefore, $\|T\| \leq\left\|T_{2}\right\|$ and similarly, $\left\|T_{1}\right\| \leq\|T\|$. The uniqueness of the solution $T$ follows by the density of polynomials in $X$ via continuity of the linear operator $T$. This ends the proof.

Our next goal is to give a result for the Markov moment problem in the space $L_{\nu}^{1}(\mathbb{R})$, where $\nu$ is a moment determinate measure on $\mathbb{R}$ having finite moments $\int_{\mathbb{R}} t^{k} d \nu$ of all orders $k \in \mathbb{N}$.

Corollary 4.3. Let $X=L_{\nu}^{1}(\mathbb{R})$, where $\nu$ is a moment determinate positive measure on $\mathbb{R}$. Assume that $Y$ is an arbitrary order complete Banach lattice and $\left(y_{n}\right)_{n \geq 0}$ is a given sequence having its terms in $Y$. Let $T_{1}$ and $T_{2}$ be two linear operators from $X$ to $Y$ such that $\mathbf{0} \leq T_{1} \leq T_{2}$ on $X_{+}$. The following statements are equivalent:

(a). There exists a unique bounded linear operator $T$ from $X$ to $Y, T_{1} \leq T \leq T_{2}$ on $X_{+},\left\|T_{1}\right\| \leq\|T\| \leq\left\|T_{2}\right\|$, such that $T\left(\varphi_{n}\right)=y_{n}$ for all $n \in \mathbb{N}$.

(b). If $J_{0} \subset \mathbb{N}$ is a finite subset and $\left\{\lambda_{j} ; j \in J_{0}\right\} \subset \mathbb{R}$ then

$$
\sum_{i, j \in J_{0}} \lambda_{i} \lambda_{j} T_{1}\left(\varphi_{i+j}\right) \leq \sum_{i, j \in J_{0}} \lambda_{i} \lambda_{j} y_{i+j} \leq \sum_{i, j \in J_{0}} \lambda_{i} \lambda_{j} T_{2}\left(\varphi_{i+j}\right) .
$$

Corollary 4.4. Let $\nu$ be a moment determinate positive Borel measure on $\mathbb{R}$, with finite moments of all orders. Assume that $h_{1}$ and $h_{2}$ are two functions in $L_{\nu}^{\infty}(\mathbb{R})$, such that $\mathbf{0} \leq h_{1} \leq h_{2}$ almost everywhere. Let $\left(y_{n}\right)_{n \geq 0}$ be a given sequence of real numbers. The following statements are equivalent:

(a). There exists $h \in L_{\nu}^{\infty}(\mathbb{R})$, such that $h_{1} \leq h \leq h_{2} \nu$-almost everywhere, $\int_{\mathbb{R}} t^{j} h(t) d \nu=y_{j}$ for all $j \in \mathbb{N}$.

(b). If $J_{0} \subset \mathbb{N}$ is a finite subset and $\left\{\lambda_{j} ; j \in J_{0}\right\} \subset \mathbb{R}$ then

$$
\sum_{i, j \in J_{0}} \lambda_{i} \lambda_{j} \int_{\mathbb{R}} t^{i+j} h_{1}(t) d \nu \leq \sum_{i, j \in J_{0}} \lambda_{i} \lambda_{j} y_{i+j} \leq \sum_{i, j \in J_{0}} \lambda_{i} \lambda_{j} \int_{\mathbb{R}} t^{i+j} h_{2}(t) d \nu
$$

In cases of Corollaries 4.3 and 4.4, we have $\mathcal{P}_{++}=\mathcal{P}_{+}$. Going further to the multidimensional case, when nonnegative polynomials are not usually expressible as sums of squares, the inclusion $\mathcal{P}_{++} \subset \mathcal{P}_{+}$is strict. In the case of $F=\mathbb{R}_{+}^{n}$ (respectively $F=\mathbb{R}^{n}$ ), $n \geq 2$, the cone $\mathcal{P}_{++}$consists of all polynomials which are sums of products of the form

$$
p_{1} \otimes \cdots \otimes p_{n}
$$

where each $p_{j}, j=1, \ldots, n$, is a nonnegative polynomial on $\mathbb{R}_{+}$(respectively on $\mathbb{R}$ ), hence is expressible by means of sums of squares of polynomials of one variable. Proceeding this way, the last conditions of (7) can be written in terms of quadratic forms (see Corollary 4.5 stated as follows).

Corollary 4.5. Let $\nu=\nu_{1} \times \cdots \times \nu_{n}, n \geq 2, \nu_{j}$ being an $M$-determinate (moment determinate) positive regular Borel measure on $\mathbb{R}, j=1, \ldots, n, X=L_{\nu}^{1}\left(\mathbb{R}^{n}\right), \varphi_{j}(t)=t^{j}, t \in \mathbb{R}^{n}, j \in \mathbb{N}^{n}$. Additionally, assume that $\nu_{j}$ has finite moments of all orders, $j=1, \ldots, n$. Let $Y$ be an order complete Banach lattice, $\left(y_{j}\right)_{j \in \mathbb{N}^{n}}$ be a given sequence of elements in $Y$, and $T_{1}, T_{2}$ be two bounded linear operators from $X$ to $Y$. The following statements are equivalent: 
(a). There exists a unique (bounded) linear operator $T: X \rightarrow Y, T\left(\varphi_{j}\right)=y_{j}, j \in \mathbb{N}^{n}, \mathbf{0} \leq T_{1} \leq T \leq T_{2}$ on $X_{+}$,

$$
\left\|T_{1}\right\| \leq\|T\| \leq\left\|T_{2}\right\| \text {. }
$$

(b). For any finite subset $J_{0} \subset \mathbb{N}^{n}$ and for any $\left\{\lambda_{j} ; j \in J_{0}\right\} \subset \mathbb{R}$, the following implication holds:

$$
\sum_{j \in J_{0}} \lambda_{j} \varphi_{j} \in \mathcal{P}_{+} \Longrightarrow \sum_{j \in J_{0}} \lambda_{j} T_{1}\left(\varphi_{j}\right) \leq \sum_{j \in J_{0}} \lambda_{j} y_{j}
$$

and for any finite subsets $J_{k} \subset \mathbb{N}, k=1, \ldots, n$, and for any $\left\{\lambda_{j_{k}}\right\}_{j_{k} \in J_{k}} \subset \mathbb{R}$, the following inequalities hold:

$$
\begin{aligned}
0 \leq & \sum_{i_{1}, j_{1} \in J_{1}}\left(\ldots\left(\sum_{i_{n}, j_{n} \in J_{n}} \lambda_{i_{1}} \lambda_{j_{1}} \cdots \lambda_{i_{n}} \lambda_{j_{n}} T_{1}\left(\varphi_{i_{1}+j_{1}, \ldots, i_{n}+j_{n}}\right)\right) \cdots\right) ; \\
& \sum_{i_{1}, j_{1} \in J_{1}}\left(\cdots\left(\sum_{i_{n}, j_{n} \in J_{n}} \lambda_{i_{1}} \lambda_{j_{1}} \cdots \lambda_{i_{n}} \lambda_{j_{n}} y_{i_{1}+j_{1}, \ldots, i_{n}+j_{n}}\right) \cdots\right) \leq \\
& \sum_{i_{1}, j_{1} \in J_{1}}\left(\cdots\left(\sum_{i_{n}, j_{n} \in J_{n}} \lambda_{i_{1}} \lambda_{j_{1}} \cdots \lambda_{i_{n}} \lambda_{j_{n}} T_{2}\left(\varphi_{i_{1}+j_{1}, . ., i_{n}+j_{n}}\right)\right) \cdots\right) .
\end{aligned}
$$

The last consequences of Theorem 4.3 refer to the Markov moment problem on $\mathbb{R}_{+}$.

Corollary 4.6. Let $X=L_{\nu}^{1}\left(\mathbb{R}_{+}\right)$, where $\nu$ is a moment determinate positive Borel measure on $\mathbb{R}_{+}$. Assume that $Y$ is an arbitrary order complete Banach lattice, and $\left(y_{n}\right)_{n \geq 0}$ is a given sequence having its terms in $Y$. Let $T_{1}$ and $T_{2}$ be two linear operators from $X$ to $Y$ such that $0 \leq T_{1} \leq T_{2}$ on $X_{+}$. As usual, we denote $\varphi_{j}(t)=t^{j}, j \in \mathbb{N}, t \in \mathbb{R}_{+}$. The following statements are equivalent:

(a). There exists a unique bounded linear operator $T$ from $X$ to $Y, T_{1} \leq T \leq T_{2}$ on $X_{+},\left\|T_{1}\right\| \leq\|T\| \leq\left\|T_{2}\right\|$, such that $T\left(\varphi_{n}\right)=y_{n}$ for all $n \in \mathbb{N}$.

(b). If $J_{0} \subset \mathbb{N}$ is a finite subset and $\left\{\lambda_{j} ; j \in J_{0}\right\} \subset \mathbb{R}$ then

$$
\sum_{i, j \in J_{0}} \lambda_{i} \lambda_{j} T_{1}\left(\varphi_{i+j+k}\right) \leq \sum_{i, j \in J_{0}} \lambda_{i} \lambda_{j} y_{i+j+k} \leq \sum_{i, j \in J_{0}} \lambda_{i} \lambda_{j} T_{2}\left(\varphi_{i+j+k}\right), k \in\{0,1\} .
$$

Corollary 4.7. Let $\nu$ be a moment determinate positive Borel measure on $\mathbb{R}_{+}$, with finite moments of all orders. Assume that $h_{1}, h_{2}$ are two functions in $L_{\nu}^{\infty}\left(\mathbb{R}_{+}\right)$, such that $\mathbf{0} \leq h_{1} \leq h_{2}$ almost everywhere. Let $\left(y_{n}\right)_{n \geq=0}$ be a given sequence of real numbers. The following statements are equivalent:

(a). There exists $h \in L_{\nu}^{\infty}\left(\mathbb{R}_{+}\right)$, such that $h_{1} \leq h \leq h_{2} \nu$-almost everywhere and $\int_{\mathbb{R}_{+}} t^{j} h(t) d \nu=y_{j}$ for all $j \in \mathbb{N}$.

(b). If $J_{0} \subset \mathbb{N}$ is a finite subset and $\left\{\lambda_{j} ; j \in J_{0}\right\} \subset \mathbb{R}$, then

$$
\sum_{i, j \in J_{0}} \lambda_{i} \lambda_{j} \int_{\mathbb{R}_{+}} t^{i+j+k} h_{1}(t) d \nu \leq \sum_{i, j \in J_{0}} \lambda_{i} \lambda_{j} y_{i+j+k} \leq \sum_{i, j \in J_{0}} \lambda_{i} \lambda_{j} \int_{\mathbb{R}_{+}} t^{i+j+k} h_{2}(t) d \nu, k \in\{0,1\}
$$

\section{On the truncated moment problem}

This section is subdivided into two parts, namely: (i) General type results and existence of solutions (ii) Perturbations of moments and the corresponding perturbations of solutions.

\section{General type results and existence of solutions}

The truncated moment problem is important in mathematics because it involves only a finite number of moments (of limited order), which are assumed to be known (or given, or measurable); therefore, it can be related to optimization problems [12] as well as to constructive methods for finding solutions [9,10,25,26] (see [25,26] for the existence of a polynomial solution). We start by recalling the truncated (reduced) Markov moment problems on a closed, bounded or unbounded subset $F$ of $\mathbb{R}^{n}$, where $n \geq 1$ is an integer. We denote by $\mathbb{R}_{d}\left[t_{1}, \ldots t_{n}\right]$ the real vector subspace of all polynomial functions $\mathcal{P}$ of $n$ real variables, with real coefficients, generated by $t^{k}=t_{1}^{k_{1}} \cdots t_{n}^{k_{n}}, k_{i} \in\{0,1, \ldots, d\}, i=1, \ldots, n$, where $d \geq 1$ is a fixed integer. The dimension of this subspace is clearly equal to $N=(d+1)^{n}$. Given a finite set $\left\{m_{k}\right\}_{0 \leq k_{i} \leq d ; i=1, \ldots, n}$ of real numbers and a 
positive Borel measure $\mu$ on $F$ with finite absolute moments of all orders smaller than or equal to $d$ (i.e., $\int_{F}|t|^{k} d \mu<\infty$ for all $k=\left(k_{1}, \ldots, k_{n}\right) \in \mathbb{N}^{n}$ with $\left.k_{i} \leq d, i=1, \ldots, n\right)$, one studies the existence and, eventually construction or approximation of a Lebesgue measurable real function $h \in L_{\mu}^{\infty}(F)$, satisfying the moment conditions

$$
\int_{F} t^{k} h(t) d \mu=m_{k}, k_{i} \leq d, i=1, \ldots, n,
$$

and $\mathbf{0} \leq h \leq b \cdot \mathbf{1}$, for some positive number $b$. An application of Theorem 3.1 and standard measure theory arguments (see [29]) lead to the following existence type results for scalar-valued solution of the truncated moment problem. Let $F \subseteq \mathbb{R}^{n}$ be a closed unbounded subset and $\mu$ be a positive regular Borel measure on $F$.

Theorem 5.1 (see [25], Theorem 2.2). Assume that all absolute moments $\int_{F}|t|^{k} d \mu<\infty$ for $k=\left(k_{1}, \ldots, k_{n}\right)$ with $k_{i} \leq d$, $i=1, \ldots, n$, are finite. Let $\left\{m_{k}\right\}_{k_{i} \leq d ; i=1, \ldots, n}$ be a given (finite) sequence of real numbers. The following statements are equivalent:

(a). There exists $h \in L_{\mu}^{\infty}(F), \mathbf{0} \leq h \leq \mathbf{1}, \quad \int_{\mathrm{F}} t^{k} h(t) d \mu=m_{k}, k_{i} \leq d, i=1, \ldots, n$.

(b). For any family of scalars $\left\{a_{k}\right\}_{k_{i} \leq d ; i=1, \ldots, n}$ the inequality

$$
\sum_{k_{i} \leq d ; i=1, \ldots, n} a_{k} \varphi_{k} \leq \varphi \in L_{\mu}^{1}(F)
$$

implies

$$
\sum_{k_{i} \leq d ; i=1, \ldots, n} a_{k} m_{k} \leq \int_{F}|\varphi(t)| d \mu=\|\varphi\|_{1, \mu} .
$$

Theorem 5.2 (see [25], Theorem 2.4). Let $p \in(1, \infty), X=L_{\mu}^{p}(F),\left\{m_{k}\right\}_{k_{i} \leq d ; i=1, \ldots, n}$ be a given (finite) sequence of real numbers, and $q$ be the conjugate of $p$. The following statements are equivalent:

(a). There exists $h \in L_{\mu}^{q}(F), 0 \leq h(t)$ a.e., $\|h\|_{q, \mu} \leq 1, \int_{F} t^{k} h(t) d \mu=m_{k}, k_{i} \leq d, i=1, \ldots, n$.

(b). For any family of scalars $\left\{a_{k}\right\}_{k_{i} \leq d ; i=1, \ldots, n}$ the inequality

$$
\sum_{k_{i} \leq d ; i=1, \ldots, n} a_{k} \varphi_{k} \leq \varphi \in L_{\mu}^{p}
$$

implies

$$
\sum_{k_{i} \leq d ; i=1, \ldots, n} a_{k} m_{k} \leq\|\varphi\|_{p, \mu}
$$

\section{Perturbations of moments and the corresponding perturbations of solutions}

Assume now that the moments $m_{k}$, where $k=\left(k_{1}, \ldots, k_{n}\right) \in \mathbb{N}_{0}^{n}, 0 \leq k_{i} \leq d, i \in\{1,2, \ldots, n\}$ are exact, determined in a training stage, when no external influence can occur. Also, assume that the moments in real time stage, denoted by $v_{k}$, where $k_{i} \leq d, i=1, \ldots, n$, can be measured, but errors may occur due to external influences. The vector subspace of polynomials involved this way has the dimension $(d+1)^{n}$. Assume now that there exists $h, w$ in $L_{\mu}^{2}(F)$ such that

$$
\int_{S} t^{k} h(t) d \mu=m_{k}, \int_{S} t^{k} w(t) d \mu=v_{k}
$$

for all multi-index $k \in \mathbb{N}_{0}^{n}$ having all components $0 \leq k_{i} \leq d, i=1, \ldots, n$. Let $\left\{e_{k}\right\}_{0 \leq k_{i} \leq d}$ be the orthogonal system of polynomials having unit norms, obtained from the system $\left\{\varphi_{k}\right\}_{0 \leq k_{i} \leq d}$ via Gram-Schmidt process, in the space $L_{\mu}^{2}(F)$. Denote by $P_{d}: L_{\mu}^{2}(F) \rightarrow \operatorname{Span}\left\{e_{k} ; k_{i} \in\{0, \ldots, d\}, i=1, \ldots, n\right\}$ the orthogonal projection. It holds that

$$
e_{k}=\sum_{l \leq k} c_{l, k} \varphi_{l}
$$

where $l \leq k$ means $l_{i} \leq k_{i}, i=1, \ldots, n$, and the coefficients $c_{l, k}$ are known from Gram-Schmidt process. It yields

$$
\left\langle P_{d}(h)-P_{d}(w), e_{k}\right\rangle=\sum_{l \leq k} c_{l, k}\left\langle P_{d}(h)-P_{d}(w), \varphi_{l}\right\rangle=\sum_{l \leq k} c_{l, k}\left(m_{l}-v_{l}\right) .
$$

Thus,

$$
\left\|P_{d}(w)-P_{d}(h)\right\|_{2, \mu}^{2}=\sum_{k_{i} \leq d}\left\langle P_{d}(h)-P_{d}(w), e_{k}\right\rangle^{2}==\sum_{k_{i} \leq d}\left(\sum_{l \leq k} c_{l, k}\left(m_{l}-v_{l}\right)\right)^{2} .
$$

Hence, one determines the integral mean of the square $\left(P_{d}(w)-P_{d}(h)\right)^{2}$ on the left hand side of (9), in terms of the squares of the errors $\left|m_{k}-v_{k}\right|, \quad\left(k_{i} \leq d\right.$ for all $\left.i \in\{1, \ldots, n\}\right)$. 


\section{Conclusion}

Some Hahn-Banach type results are reviewed and their recently published applications are discussed. Also, their direct applications to concrete spaces are stated and some of them are proved. One of the applications of Theorem 3.2 consists of proving an unexpected sandwich theorem (see Theorems 3.6 and 3.7). An example of a Mazur-Orlicz type theorem on concrete spaces is emphasized in Theorem 3.5. On the other hand, existence and uniqueness of the solutions for the classical Markov full moment problem are emphasized, by means of the polynomial approximation on unbounded subsets, accompanied by the extension result stated in Theorem 2.1. In the last part of the paper (Section 5), a few aspects of the truncated moment problem are discussed.

\section{Acknowledgement}

The author would like to thank the anonymous referees for their comments and suggestions that lead to the improvement of the presentation of this paper.

\section{References}

[1] N. I. Akhiezer, The Classical Moment Problem and Some Related Questions in Analysis, Oliver and Boyd, London, 1965.

[2] C. Ambrozie, O. Olteanu, A sandwich theorem, the moment problem, finite-simplicial sets and some inequalities, Rev. Roumaine Math. Pures Appl. 49 (2004) 189-210.

[3] C. Berg, J. P. Christensen, C. U. Jensen, A remark on the multidimensional moment problem, Math. Ann. 243 (1979) $163-169$.

[4] C. Berg, J. P. Christensen, P. Ressel, Harmonic Analysis on Semigroups: Theory of Positive Definite and Related Functions, Springer, New York, 1984.

[5] G. Cassier, Problèmes des moments sur un compact de $\mathbb{R}^{\mathrm{n}}$ et décomposition des polynômes à plusieurs variables, J. Funct. Anal. 58 (1984) $254-266$.

[6] G. Choquet, Le problème des moments, In Séminaire d'Initiation à l'Analise, Institut H. Poincaré, Paris, 1962.

[7] R. Cristescu, Ordered Vector Spaces and Linear Operator, Editura Academiei, Bucharest; Abacus Press, Abacus Press, Tunbridge Wells, 1976.

[8] B. Fuglede, The multidimensional moment problem, Expo. Math. 1 (1983) 47-65.

[9] L. Gosse, O. Runborg, Resolution of the finite Markov moment problem, C. R. Math. Acad. Sci. Paris 341 (2005) 775-780.

[10] L. Gosse, O. Runborg, Existence, uniqueness, and a constructive solution algorithm for a class of finite Markov moment problems, SIAM J. Appl. Math. 68 (2008) 1618-1640.

[11] E. K. Haviland, On the momentum problem for distributions in more than one dimension, Amer. J. Math. 58 (1936) $164-168$.

[12] P. L. Inverardi, A. Tagliani, Stieltjies and Hamburger reduced moment problem when MaxEnt solution does not exist, Mathematics $\mathbf{9}$ (2021) \#309.

[13] M. G. Krein, A. A. Nudelman, Markov Moment Problem and Extremal Problems, Amer. Math. Soc., Providence, 1977.

[14] S. S. Kutateladze, Convex operators, Russian Math. Surveys 34 (1979) 181-214.

[15] L. Lemnete, An operator-valued moment problem, Proc. Amer. Math. Soc. 112 (1991) 1023-1028.

[16] L. Lemnete-Ninulescu, A. Zlătescu, Some new aspects of the L-moment problem, Rev. Roumaine Math. Pures Appl. 55 (2010) 197-204.

[17] C. P. Niculescu, O. Olteanu, From the Hahn-Banach extension theorem to the isotonicity of convex functions and the majorization theory, Rev. $R$. Acad. Cienc. Exactas Fis. Nat. RACSAM 114 (2020) \#171.

[18] C. P. Niculescu, L. E. Persson, Convex Functions and Their Applications: A Contemporary Approach, 2nd Edition, Springer, New York, 2018.

[19] O. Olteanu, Convexité et prolongement d'opérateurs linéaires, (Convexity and extension of linear operators), C. R. Acad. Sci. Paris Série A 286 (1978) 511-514.

[20] O. Olteanu, Théorèmes de prolongement d'opérateurs linéaires, (Theorems on extension of linear operators), Rev. Roumaine Math. Pures Appl. 28 (1983) 953-983.

[21] O. Olteanu, Application de théorèmes de prolongement d'opérateurs linéaires au problème des moments e à une generalization d'un théorème de Mazur-Orlicz, (Applications of theorems on extension of linear operators to the moment problem and to a generalization of Mazur-Orlicz theorem), C. R. Acad. Sci. Paris Série I 313 (1991) 739-742.

[22] O. Olteanu, New results on Markov moment problem, Int. J. Anal. 2013 (2013) \#901318.

[23] O. Olteanu, From Hahn-Banach type theorems to the Markov moment problem, sandwich theorems and further applications, Mathematics 8 (2020) $\# 1328$.

[24] O. Olteanu, Polynomial approximation on unbounded subsets, Markov moment problem and other applications, Mathematics 8 (2020) \#1654.

[25] O. Olteanu, On truncated and full classical Markov moment problems, Contrib. Math. 3 (2021) 29-36.

[26] O. Olteanu, On Markov moment problem and related results, Symmetry 13 (2021) \#986.

[27] G. Păltineanu, I. Bucur, Some density theorems in the set of continuous functions with values in the unit interval, Mediterr. J. Math. 14 (2017) \#44.

[28] M. Putinar, Positive polynomials on compact semi-algebraic sets, Indiana Univ. Math. J. 42 (1993) 969-984.

[29] W. Rudin, Real and Complex Analysis, 3rd Edition, McGraw-Hill, Singapore, 1987.

[30] K. Schmüdgen, The K-moment problem for compact semi-algebraic sets, Math. Ann. 289 (1991) 203-206.

[31] K. Schmüdgen, The Moment Problem, Springer, Cham, 2017.

[32] J. M. Stoyanov, G. D. Lin, P. Kopanov, New checkable conditions for moment determinacy of probability distributions, Theory Probab. Appl. 65 (2020) 497-509.

[33] F. H. Vasilescu, Spectral measures and moment problems, In: A. Gheondea, M. Şabac (Eds.), Spectral Analysis and its Applications, Ion Colojoară Anniversary Volume, Theta, Bucharest, 2003, pp. 173-215. 